\title{
Everything in its right place
}

Clever design strategies enable researchers to harness nonspecific interactions to achieve highly specific arrangements of nucleic acid nanostructures.

For the aspiring DNA craftsman, basepairing might seem like the natural 'glue' to hold your constructions together. Indeed, the powerful natural attraction of adenine to thymine and guanine to cytosine has enabled scientists to engineer sophisticated self-assembling nanostructures from hybridized strands of nucleic acids.

However, 'DNA origami' pioneer Paul Rothemund and graduate student Sungwook Woo have now found the potential for building bigger and better multi-origami assemblies based on the strong and sequenceindependent interactions between blunt DNA ends.

The stacking interactions between adjacent base pairs are known to make a vital contribution to the stabilization of DNA duplexes, but the two California Institute of Technology researchers were particularly inspired by an unexpected outcome from Rothemund's early work with DNA origami rectangles. "They readily stacked against each other, and almost all the rectangles found were in chains," says Woo of the rectangles, "but they did not have any order." This raised the question of whether it might be possible to design DNA structures that interact orthogonally, such that each segment only associates with a specific partner in a particular orientation.

Each DNA origami piece consists of a long circular scaffold molecule that bends into shape through base-pairing interactions with many precisely positioned 'staple' oligonucleotides. Through careful computational design, Woo and Rothemund constructed multiple components with different configurations of blunt ends exposed at the edges and tested two different strategies for directing origami-origami interactions.

In the binary approach, each shape presents a particular arrangement of projecting blunt-ended 'patches' separated by floppy DNA loops, with interaction dependent on the proper alignment of these patches between each origami pair. The binary approach is highly flexible, as the configuration of patches and loops at each edge can readily be altered by adjusting the hybridization of the origami using different staple

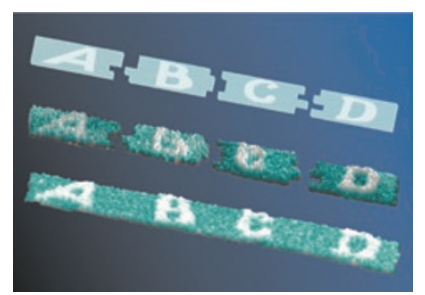

A shape-based approach to origami assembly. The pieces plug directly into one another via matching projections and concavities. Origami molecules are labeled via hybridization of additional DNA hairpin molecules, which can be detected by atomic force microscopy. Image courtesy of S. Woo and P. Rothemund.

combinations to achieve what Woo describes as "reprogramming".

In the second, shape-based approach, the researchers designed origami pieces that present unique configurations of projections and recesses at their edges, such that the components assemble like puzzle pieces. As a test of this approach, Rothemund and Woo designed four discrete segment types that preferentially interacted in a particular order.

Rothemund points out that this work demonstrates how the nonspecific stacking interactions that have given the DNA origami community such headaches in the past can be harnessed to good effect. "If you are careful, you can harness the power of blunt-end stacking interactions to make larger objects out of DNA in a very simple and easy way," he says, "converting something that used to be considered a bug into a feature."

This could also prove a boon to the construction of more sophisticated DNA-based devices with multiple moving parts. Such systems necessarily require the building and breaking of connections between DNA components, but the strength of the bond between hybridized nucleotides can establish a high energetic barrier for this process. "We think that we will be able to design stacking bonds in which the parts of a nanomachine can both self-assemble and slide freely past each other when the time comes to run the machine," says Rothemund.

\section{Michael Eisenstein}

\section{RESEARCH PAPERS}

Woo, S. \& Rothemund, P.W.K. Programmable molecular recognition based on the geometry of DNA nanostructures. Nat. Chem. 3, 620-627 (2011). 